\title{
Standard Heterosis of Maize (Zea Mays L.) Genotypes for Grain Yield and Yield Related Traits in Mid-Altitude Agro-Ecology of Ethiopia
}

\author{
Mamud Aman Tello \\ Maize Breeder, Wondo Genet Agricultural Research Center, Hawassa Maize, Ethiopian Institute of Agricultural \\ Research, Addis Ababa, Ethiopia
}

\begin{abstract}
A line $\mathrm{x}$ tester analysis involving sixty four test-crosses generated by crossing 32 elite maize inbred lines with two testers and two standard checks was evaluated for yield and yield related traits in $6 \times 11$ alpha lattice design replicated twice during 2017 cropping season at Bako National Maize Research Center. The study was designed to estimate the amount of standard heterosis of the hybrids for grain yield and yield related traits. From this study, considerable standard heterosis for all traits over both commercial checks was manifested. For grain yield, the highest standard heterosis was recorded for L17xT1 (61.75\%) and (41.46\%) over BH546 and BH547, respectively, indicating the presence of substantial heterotic potential that could be exploited in maize breeding program and possibility of developing desirable cross combinations through crossing of inbred lines with desirable traits of interest. Cross L3xT2 manifested negative standard heterosis over BH546 and BH547 for days to anthesis, silking and maturity that indicate earliness of the crosses in maturity as compared to both standard checks. Similarly, most of the crosses showed positive standard heterosis for biomass yield, harvest index, ear per plant, ear length, ear diameter, rows per ear, kernel per rows and thousand kernel weights over both commercial checks. In general, information from this study could be valuable for researchers who intend to develop high yielding maize varieties. Keywords: Maize, Standard Heterosis, Grain Yield.
\end{abstract}

DOI: $10.7176 / \mathrm{JBAH} / 11-4-03$

Publication date: February $28^{\text {th }} 2021$

\section{INTRODUCTION}

Maize (Zea mays L.) is one of the most important crops in the world agricultural economy. It is a vital cereal crop for global food security, as its importance is seen in the wide economic uses ranging from direct human consumption to processing into other food materials, animal feeds, and industrial products. It ranked first among the cereal with an annual global production of more than one billion metric tons (FAOSTAT, 2016). It is a major staple food crop grown in diverse agro-ecological zones and farming systems and consumed by people with varying food preferences and socio-economic backgrounds in sub Saharan Africa (SSA).

In Ethiopia, maize is one of the most important cereal crops grown, mainly for food security. The total annual production and productivity exceed all other cereal crops except tef in area coverage (Mosisa et al., 2012). Despite the large area under maize, the national average yield of maize is about $3.9 \mathrm{t} / \mathrm{ha}$ (CSA, 2018). This is by far below the world's average yield which is about $5.4 \mathrm{t} / \mathrm{ha}$ (FAO, 2016). Besides many factors, unavailability of suitable maize varieties is one of the possible reasons responsible for such yield gap. To increase production of maize, efforts are required to be made to develop hybrids with high yielding potential. In all breeding schemes except line breeding, heterosis is a major factor for yield increase (Schnell, 1982). Thus, this study was designed to determine the magnitude of standard heterosis for yield and yield related traits of maize inbred lines.

\section{MATERIALS AND METHODS \\ Experimental Site}

This experiment was conducted at Bako National Maize Research Center during the 2017 main cropping season. The area is located in East Wollega Zone of the Oromia Regional State, Western Ethiopia at about $250 \mathrm{~km}$ west of Addis Ababa, the capital city of the country. It lies between $9^{0} 6$ ' North latitude and $37^{0} 09^{\prime}$ east longitude in the sub-humid agro-ecology, with an altitude of 1650 meters above sea level. The mean annual rainfall of the study area during the study season was $1316.7 \mathrm{~mm}$. The climate of the area is characterized as sub-humid with mean monthly maximum and minimum temperature of $24.45^{\circ} \mathrm{C}$ and $19.70^{\circ} \mathrm{C}$; respectively. Sixty percent $(60 \%)$ of the soil of BNMRC is reddish brown in colour with clay and loam texture.

\section{The Experimental Materials}

Sixty-four (64) test-crosses produced by crossing 32 elite inbred lines to two testers (ILO'00E-1-9-1-1-1-1-1-\# and PO'00E-3-2-1-2-1-\#-\#), and two standard checks (BH546 and BH547) were used for this study. Tester, PO'00E3-2-1-2-1-\#-\# belongs to heterotic group A and ILO'00E-1-9-1-1-1-1-1-\# has been identified as heterotic group B. All the lines and testers were developed at Bako National Maize Research Center, and the standard checks were 
released by the same center.

Table 1. Descriptions of the lines, testers and checks used for the study at Bako during 2017

\begin{tabular}{clcl}
\hline Line code & \multicolumn{1}{c}{ Pedigree } & Line code & \multicolumn{1}{c}{ Pedigree } \\
\hline L1 & S719(F2)-4-2-1-1 & L20 & P12W(F2)-162-1-1-1 \\
L2 & S719(F2)-25-1-1-1 & L21 & P12W(F2)-162-1-1-2 \\
L3 & P63(F2)-27-2-1-1 & L22 & P12W(F2)-194-1-1-2 \\
L4 & P63(F2)-31-1-1-1 & L23 & P12W(F2)-207-1-1-1 \\
L5 & P63(F2)-31-1-1-2 & L24 & SY312/442(F2)-36-2-1-1 \\
L6 & P12W(F2)-4-1-1-1 & L25 & SY312/442(F2)-84-2-1-1 \\
L7 & P12W(F2)-14-1-1-1 & L26 & SY312/442(F2)-231-1-1-1 \\
L8 & P12W(F2)-24-1-1-1 & L27 & SY312/442(F2)-234-2-1-1 \\
L9 & P12W(F2)-24-2-1-1 & L28 & SY395/202(F2)-28-1-1-1 \\
L10 & P12W(F2)-24-2-2-1 & L29 & SY395/202(F2)-66-1-1-1 \\
L11 & P12W(F2)-34-1-1-2 & L30 & P8M-91(F2)-12-1-1-1-1 \\
L12 & P12W(F2)-80-1-2-1 & L31 & P8M-91(F2)-37-2-1-1-1 \\
L13 & P12W(F2)-82-1-1-1 & L32 & P8M-91(F2)-37-2-2-1-1 \\
L14 & P12W(F2)-95-1-1-1 & & Testers \\
L15 & P12W(F2)-95-2-1-1 & T1 & ILO'00E-1-9-1-1-1-1-1-\# (B) \\
L16 & P12W(F2)-96-1-2-1 & T2 & PO'00E-3-2-1-2-1-\#-\# (A) \\
L17 & P12W(F2)-141-2-1-1 & & Standard Checks \\
L18 & P12W(F2)-161-1-2-1 & Check 1 & BH546 \\
L19 & P12W(F2)-161-1-2-2 & Check2 & BH547 \\
\hline
\end{tabular}

\section{Experimental Design and Field Management}

The experiment was conducted in a 6 x11 alpha-lattice design with two replications. Each of the 11 incomplete blocks in replication was comprised of 6 plots having 5.1-meter row length with the spacing of $0.75 \mathrm{~m}$ between rows and $0.30 \mathrm{~m}$ between plants. All cultural practices such as land preparation, weeding, disease and insect pest control were done manually as per required.

\section{Data Collected}

Grain yield $(\mathbf{G Y})$ : The total grain yield in $\mathrm{kg}$ per harvested plot and adjusted to $12.5 \%$ moisture level. Days to anthesis (DA): Number of days from planting to when $50 \%$ of the plant in a plot shed pollen. Days to silking (DS): Number of days from planting to when $50 \%$ of the plants in a plot produced $2-3 \mathrm{~cm}$ long silk. Days to maturity (DM): number of days from planting to when $50 \%$ of the kernels on the cob showed black layer on the tip the kernel where it attaches to the cob. Biomass yield (BY): Total aboveground biomass yield after oven drying to a constant weight in tons per hectare obtained from each plot at harvest. Harvest Index (HI): The ratio of dried grain weight per ha adjusted to $12.5 \%$ moisture content to the dried total aboveground biomass weight per ha. Ear length (EL): Average lengths of five randomly selected ears were measured in $\mathrm{cm}$ from the base to the tip of the ear. Ear diameter (ED): Average diameters of five randomly selected ears were measured in $\mathrm{cm}$ at mid-section along the length of the ear using a calliper. Number of ears per plant (EPP): Total number of harvested ears in each plot divided by the total number of stand count at harvest. Number of kernel rows per ear (KRPE): Total numbers of kernel rows of the ear were counted from five randomly taken ears and the average value was used as kernel rows per ear. Number of kernels per row (KPR): Number of kernels in each row from five randomly taken ears and the average values were recorded as kernels per row. Thousand kernel weight (TKW): 1000 randomly taken kernels were weighed from each plot using sensitive balance and was adjusted to $12.5 \%$ moisture level.

\section{Data Analysis}

Analysis of Variance (ANOVA)

The data collected for all yield and yield related traits were analyzed using PROC MIXED procedure in SAS (SAS, 2004).

\section{Estimation of Standard Heterosis}

Percent standard heterosis was calculated for traits showed statistically significant differences among genotypes as suggested by Falconer and Mackay (1996). This was computed as percentage increase or decrease of the cross performances over the best standard checks as follows. BH-546 and BH-547 were used as standard checks.

$$
\mathrm{SH}(\%)=\frac{F 1-\mathrm{sV}}{\mathrm{SV}} \mathrm{X} 100
$$

Where $\mathrm{F} 1=$ Mean value of the cross

$\mathrm{SV}=$ Mean value of the standard variety 
$\mathrm{SH}=$ standard heterosis

Test of significance for percent heterosis was made by using the t-test. The standard errors of the difference for heterosis and $\mathrm{t}$-value were computed as follows:

$$
\begin{aligned}
& \mathrm{t}(\text { standard cross })=\frac{F 1-S V}{S E(d)} \\
& \mathrm{t}(\text { economic })=\mathrm{SH} / \mathrm{SE} \\
& \mathrm{SE}(\mathrm{d})=(2 \mathrm{ME} / \mathrm{r})^{1 / 2}
\end{aligned}
$$

Where, SE (d) is standard error of the difference

$$
\begin{aligned}
& \mathrm{Me}=\text { error mean square } \\
& \mathrm{r}=\text { the number of replications and } \\
& \mathrm{SH}=\text { standard heterosis }
\end{aligned}
$$

The computed $t$ value tested against the t-value at the degree of freedom of error.

\section{RESULTS AND DISCUSSION}

Analysis of Variance (ANOVA)

Analysis of variance was conducted for all the traits and it showed that mean squares due to genotypes were

\begin{tabular}{|c|c|c|c|}
\hline \multirow{3}{*}{ Characters } & \multicolumn{3}{|c|}{ Mean squares } \\
\hline & Replication & Genotypes & Error $(\mathrm{E})$ \\
\hline & $(\mathrm{df}=1)$ & $(\mathrm{df}=65)$ & $(\mathrm{df}=45)$ \\
\hline GY & $19.334 * *$ & $2.689 * *$ & 1.493 \\
\hline $\mathrm{DA}$ & 0.371 & $9.050 * *$ & 1.722 \\
\hline DS & 2.189 & $11.828 * *$ & 5.444 \\
\hline $\mathrm{DM}$ & 1.485 & $36.203 * *$ & 6.890 \\
\hline BY & 12.583 & $46.451 * *$ & 11.915 \\
\hline HI & $136.97^{*}$ & $69.428 * *$ & 27.504 \\
\hline EL & $16.112^{* *}$ & $3.262 * *$ & 0.708 \\
\hline ED & $0.275^{* *}$ & $0.109 * *$ & 0.028 \\
\hline NEPP & 0.0127 & $0.043 * *$ & 0.017 \\
\hline NRPE & 2.537 & $3.215^{*}$ & 1.992 \\
\hline NKPR & $79.826^{* *}$ & $45.243 *$ & 25.536 \\
\hline TKW & $14622.4 * *$ & $3251.580 * *$ & 884.646 \\
\hline
\end{tabular}
significant $(\mathrm{P}<0.01$ or $\mathrm{P}<0.05)$ for all traits (Table 2$)$. The significant mean squares due to genotypes for all the traits studied indicated the existence of variability among the genotypes, which could be exploited for the future improvement of the traits. The current finding is in line with the findings of (Shushay, 2014).

Table 2. Mean squares due to genotypes and errors for grain yield and yield related traits of maize evaluated at Bako in 2017.

$*$ and $* *=$ significant at 0.05 and 0.01 probability level, respectively

$\mathrm{df}=$ degrees of freedom

$\mathrm{DA}=$ Days to Anthesis, DS = Days to Silking, DM = Days to Maturity, ED = Ear Diameter, EL = Ear Length, $\mathrm{EPP}=$ Ear per Plant, GY = Grain Yield, HI = Harvest Index, BY = biomass yield, KPR = Kernel per Row, RPE = Rows per Ear and TKW = Thousand Kernel Weight.

\section{Standard Heterosis}

The estimates of standard heterosis over the standard checks (BH546 and BH547) were computed for grain yield and yield related traits that showed significant differences among genotypes. Among the 64 crosses, 12 crosses exhibited positive and significant or highly significant heterosis over BH546 for grain yield, whereas only 5 crosses exhibited positive and significant or highly significant heterosis over BH547 for the same trait (Table 3). Standard heterosis for this trait ranged from $61.75 \%$ for L17xT1 to $38.35 \%$ for L11xT2. Crosses L17xT1 (61.75 \%), L10xT2 (56.49\%), L14xT1 (51.53\%), L3xT2 (52.89\%), L10xT1(50.23\%) and L20xT1(47.49\%) exhibited positive and 
highly significant $(\mathrm{P}<0.01)$ standard heterosis over BH546, while crosses L19xT2 $(42.10 \%)$, L2xT2 (41.16\%), L5xT2(40.44\%), L27xT1(39.22\%), L8xT1(39.07\%) and L11xT2 (38.35\%) showed positive and significant $(\mathrm{P}<0.05)$ standard heterosis over BH546 for grain yield. Similarly, the standard heterosis over BH547 varied from $41.46 \%$ for L17xT1 to $31.11 \%$ for L10xT1. For this trait, only cross L17xT1 (41.46\%) showed positive and highly significant $(\mathrm{P}<0.01)$ standard heterosis over BH547 whereas crosses L10xT2 (36.57), L3xT2 (33.43\%),L14xT1 $(32.24 \%)$ and L10xT1(31.11\%) showed positive and significant $(\mathrm{P}<0.05)$ standard heterosis over BH547. Positive and significant heterosis is advantageous as it indicates increased yield over the existing standard check. For grain yield, larger number of the crosses showed positive and significant standard heterosis over BH546 than BH547, indicating that BH547 was the highest yielding standard check. In agreement with the current finding, the expression of grain yield heterosis above the standard check in maize has been reported by several investigators (Berhanu, 2009; Chandana, 2013; Shushay,2014 and Mesenbet et al., 2016).

For days to anthesis, standard heterosis over BH546 ranged from $-6.47 \%$ for L3xT2, L16xT2 and L24xT2 to $10.79 \%$ for L32xT1. Regarding standard heterosis over BH547 for days to anthesis, 24 crosses showed negative and significant to highly significant heterosis while only 2 crosses (L32xT1and L32xT2) showed positive and significant to highly significant heterosis for this trait. Standard heterosis over BH547 for days to anthesis ranged from $-8.45 \%$ to $8.45 \%$.

For days to silking, 4 crosses (L1xT1, L3xT2, L15xT2 and L16xT2) showed negative and significant to highly significant standard heterosis over both standard checks (BH546 and BH547), except cross L32xT1(8.39\% and 9.15\%) which showed positive and significant standard heterosis over BH546 and BH547 respectively. Standard heterosis for this trait ranged from $-15.38 \%$ to $9.15 \%$. Negative and significant standard heterosis is desirable direction as it indicates earlier anthesis and silking of the crosses than the standard check and the reverse is true for the crosses with positive and significant standard heterosis for both traits.

The estimates of standard heterosis for days to maturity over BH546 ranged from $-6.47 \%$ (L3 xT2) to $10.79 \%$ (L32 x T1). Out of 64 crosses studied for this trait, 18 of these crosses showed negative and positive significant to highly significant heterosis over BH546 (Table 3). Standard heterosis for days to maturity over BH547, ranged from $-8.45 \%$ for L3xT2, L16xT2, and L24xT2 to 8.45\% for L32xT1. 24 crosses exhibited negative and significant to highly significant heterosis while only 2 crosses (L32xT1 and L32xT2) showed positive and highly significant $(\mathrm{P}<0.01)$ and significant $(\mathrm{P}<0.05)$ standard heterosis for the trait respectively.

The extent of standard heterosis for biomass yield over BH546 varied from $109.27 \%$ (L32xT2) to $-44.21 \%$ (L29 x T2). Cross L32x T2 also showed positive and highly significant standard heterosis for biomass yield over BH547 (Table 3). The estimates of standard heterosis for biomass yield over BH547 varied from 167.26\% for L $32 x T 2$ to $37.08 \%$ for $\mathrm{L} 15 \mathrm{xT} 1$. In conformity with the current results, both positive and negative standard heterosis for biomass yield has been reported by shushay (2014).

With respect to harvest index, 32 crosses exhibited positive and significant to highly significant standard heterosis over BH546 and it ranged from $115.43 \%$ for L10x T2 to 39.35\% for L23x T1. This indicated that these crosses were the best as compared to standard check in order to increase harvest index. The standard heterosis computed for this trait over BH547 showed that only cross L10xT2 (54.51\%) showed positive and highly significant standard heterosis while Cross L32xT2 (-36.00\%) and L32xT1 (-33.83\%) showed negative and highly significant to significant standard heterosis for this trait over BH547 respectively. The standard heterosis for harvest index over BH547 varied from $54.51 \%$ for L10xT2 to $-36.00 \%$ for L32xT2 (Table 3). An agreement with the current finding Berhanu, (2009) in his study on heterosis and combining ability for yield, yield related parameters and stover quality traits for feed in maize adapted to the mid-altitude agro-ecology of Ethiopia and reported both positive and negative significant heterosis for harvest index.

Table 3. Standard heterosis of 64 crosses over BH546 and BH547 for grain yield and yield related traits evaluated at Bako in 2017.

\begin{tabular}{|c|c|c|c|c|c|c|c|c|c|c|c|c|}
\hline Code & GY & & DA & & DS & & DM & & $\mathrm{BY}$ & & $\mathrm{HI}$ & \\
\hline & BH546 & BH547 & BH546 & BH547 & BH546 & BH547 & BH546 & BH547 & BH546 & BH547 & BH546 & BH547 \\
\hline L1xT1 & 21.58 & 6.11 & 3.60 & 1.41 & $-15.38 * *$ & $-14.79 * *$ & 3.60 & 1.41 & -10.62 & 14.15 & 30.11 & -6.68 \\
\hline L1xT2 & 24.17 & 8.37 & -1.44 & -3.52 & -3.50 & -2.82 & -1.44 & -3.52 & -13.71 & 10.21 & 37.04 & -1.71 \\
\hline L2xT1 & 20.36 & 5.04 & $5.76^{* *}$ & 3.52 & 3.50 & 4.23 & $5.76^{* *}$ & 3.52 & -15.25 & 8.23 & 35.62 & -2.73 \\
\hline L2xT2 & $41.16^{*}$ & 23.19 & 3.60 & 1.41 & 2.10 & 2.82 & 3.60 & 1.41 & -5.98 & 20.07 & $43.09 *$ & 2.63 \\
\hline L $3 \times T 1$ & 0.42 & -12.36 & 1.44 & -0.70 & -0.70 & 0.00 & 1.44 & -0.70 & -26.64 & -6.31 & 30.11 & -6.68 \\
\hline L3xT2 & $52.89^{* *}$ & $33.43^{*}$ & $-6.47 * *$ & $-8.45^{* *}$ & $-7.69 *$ & $-7.04 *$ & $-6.47 * *$ & $-8.45^{* *}$ & 1.54 & 29.68 & $40.77 *$ & 0.97 \\
\hline L4xT1 & -19.31 & -29.58 & -3.60 & $-5.63 * *$ & -4.90 & -4.23 & -3.60 & $-5.63 * *$ & $-31.66^{*}$ & -12.72 & 14.11 & -18.15 \\
\hline L4xT2 & 3.73 & -9.47 & $-4.32 *$ & $-6.34 * *$ & -4.90 & -4.23 & $-4.32 *$ & $-6.34 * *$ & $-36.49 * *$ & -18.89 & $55.71 * *$ & 11.68 \\
\hline L5xT1 & 15.82 & 1.08 & 2.88 & 0.70 & 0.70 & 1.41 & 2.88 & 0.70 & -15.06 & 8.48 & 29.22 & -7.32 \\
\hline L5xT2 & $40.44 *$ & 22.57 & -1.44 & -3.52 & -2.80 & -2.11 & -1.44 & -3.52 & -15.06 & 8.48 & $57.66^{* *}$ & 13.08 \\
\hline L6xT1 & -5.85 & -17.83 & $5.76^{* *}$ & 3.52 & 3.50 & 4.23 & $5.76 * *$ & 3.52 & -15.64 & 7.74 & 6.65 & -23.51 \\
\hline L6xT2 & 17.91 & 2.90 & -0.72 & -2.82 & -2.80 & -2.11 & -0.72 & -2.82 & -23.55 & -2.37 & $48.95^{*}$ & 6.83 \\
\hline L7xT1 & 22.23 & 6.67 & 0.72 & -1.41 & -2.10 & -1.41 & 0.72 & -1.41 & -2.90 & 24.01 & 21.58 & -12.80 \\
\hline L7xT2 & 23.45 & 7.74 & -1.44 & -3.52 & -3.50 & -2.82 & -1.44 & -3.52 & -20.46 & 1.58 & $47.88^{*}$ & 6.07 \\
\hline L8xT1 & $39.07 *$ & 21.37 & 1.44 & -0.70 & -0.70 & 0.00 & 1.44 & -0.70 & 0.00 & 27.71 & 32.42 & -5.02 \\
\hline L8xT2 & 26.33 & 10.25 & -1.44 & -3.52 & -4.20 & -3.52 & -1.44 & -3.52 & -1.35 & 25.99 & 24.42 & -10.76 \\
\hline L9xT1 & 13.23 & -1.18 & 1.44 & -0.70 & -1.40 & -0.70 & 1.44 & -0.70 & -0.39 & 27.22 & 8.96 & -21.85 \\
\hline L9xT2 & 27.20 & 11.01 & -3.60 & $-5.63^{* *}$ & -5.59 & -4.93 & -3.60 & $-5.63 * *$ & -21.43 & 0.35 & $54.46^{* *}$ & 10.79 \\
\hline
\end{tabular}


Table 3. Continued...

\begin{tabular}{|c|c|c|c|c|c|c|c|c|c|c|c|c|}
\hline Code & GY & & DA & & DS & & DM & & BY & & $\mathrm{HI}$ & \\
\hline & BH546 & BH547 & BH546 & BH547 & BH546 & BH547 & BH546 & BH547 & BH546 & BH547 & BH546 & BH547 \\
\hline L10xT1 & $50.23 * *$ & $31.11^{*}$ & -0.72 & -2.82 & -3.50 & -2.82 & -0.72 & -2.82 & 0.97 & 28.94 & $42.20 *$ & 1.99 \\
\hline L10xT2 & $56.49 * *$ & $36.57^{*}$ & -2.88 & $-4.93 *$ & -5.59 & -4.93 & -2.88 & $-4.93 *$ & $-28.96 *$ & -9.27 & $115.43 * *$ & $54.51 * *$ \\
\hline L11xT1 & 30.94 & 14.27 & 1.44 & -0.70 & -1.40 & -0.70 & 1.44 & -0.70 & -12.93 & 11.19 & $44.33 * *$ & 3.52 \\
\hline L11xT2 & $38.35^{*}$ & 20.74 & -0.72 & -2.82 & -2.80 & -2.11 & -0.72 & -2.82 & -8.88 & 16.37 & $45.22 *$ & 4.16 \\
\hline L12xT1 & 34.54 & 17.41 & 0.00 & -2.11 & -2.10 & -1.41 & 0.00 & -2.11 & 1.16 & 29.19 & 27.44 & -8.59 \\
\hline L12xT2 & 14.81 & 0.20 & -2.88 & $-4.93 *$ & -4.90 & -4.23 & -2.88 & $-4.93 *$ & -24.90 & -4.09 & $45.04 *$ & 4.03 \\
\hline L13xT1 & 28.35 & 12.01 & -2.88 & $-4.93 *$ & -4.90 & -4.23 & -2.88 & $-4.93 *$ & -8.49 & 16.86 & 33.31 & -4.39 \\
\hline L13xT2 & 14.45 & -0.11 & -3.60 & $-5.63 * *$ & -4.90 & -4.23 & -3.60 & $-5.63 * *$ & $-28.76^{*}$ & -9.02 & $54.28 * *$ & 10.66 \\
\hline L14xT1 & $51.53 * *$ & $32.24^{*}$ & -1.44 & -3.52 & -4.20 & -3.52 & -1.44 & -3.52 & -6.95 & 18.84 & $54.11^{* *}$ & 10.53 \\
\hline L14xT2 & 20.64 & 5.29 & -0.72 & -2.82 & -2.80 & -2.11 & -0.72 & -2.82 & -26.06 & -5.57 & $55.17 * *$ & 11.30 \\
\hline L15xT1 & 28.35 & 12.01 & -0.72 & -2.82 & -3.50 & -2.82 & -0.72 & -2.82 & 7.34 & $37.08^{*}$ & 14.11 & -18.15 \\
\hline L15xT2 & -4.05 & -16.26 & $-4.32 *$ & $-6.34 * *$ & $-6.99 *$ & $-6.34 *$ & $-4.32^{*}$ & $-6.34 * *$ & -24.71 & -3.85 & 26.20 & -9.48 \\
\hline L16xT1 & 25.25 & 9.31 & 2.16 & 0.00 & -0.70 & 0.00 & 2.16 & 0.00 & -14.67 & 8.97 & $39.89^{*}$ & 0.33 \\
\hline L16xT2 & 19.64 & 4.41 & $-6.47 * *$ & $-8.45 * *$ & $-7.69^{*}$ & $-7.04 *$ & $-6.47 * *$ & $-8.45^{* *}$ & $-27.99 *$ & -8.04 & $58.37 * *$ & 13.59 \\
\hline L17xT1 & $61.75^{* *}$ & $41.16^{* *}$ & 0.72 & -1.41 & -0.70 & 0.00 & 0.72 & -1.41 & 19.11 & $52.12 * *$ & 29.40 & -7.19 \\
\hline L17xT2 & 4.95 & -8.41 & -3.60 & $-5.63 * *$ & -4.90 & -4.23 & -3.60 & $-5.63 * *$ & -18.92 & 3.55 & 27.62 & -8.47 \\
\hline L18xT1 & 26.04 & 10.00 & -1.44 & -3.52 & -3.50 & -2.82 & -1.44 & -3.52 & -16.22 & 7.00 & $43.80^{*}$ & 3.14 \\
\hline L18xT2 & 28.92 & 12.51 & $-4.32 *$ & $-6.34 * *$ & -4.20 & -3.52 & $-4.32 *$ & $-6.34 * *$ & -20.27 & 1.82 & $53.57 * *$ & 10.15 \\
\hline
\end{tabular}

Table 3. Continued...

\begin{tabular}{|c|c|c|c|c|c|c|c|c|c|c|c|c|}
\hline Code & GY & & $\mathrm{DA}$ & & DS & & DM & & BY & & $\mathrm{HI}$ & \\
\hline & BH546 & BH547 & BH546 & BH547 & BH546 & BH547 & BH546 & BH547 & BH546 & BH547 & BH546 & BH547 \\
\hline L19xT1 & 29.86 & 13.33 & -2.88 & $-4.93 * *$ & -3.50 & -2.82 & -2.88 & $-4.93 * *$ & -4.83 & 21.55 & 30.29 & -6.55 \\
\hline L19xT2 & $42.10^{*}$ & 24.01 & $-4.32 *$ & $-6.34 * *$ & -3.50 & -2.82 & $-4.32 *$ & $-6.34 * *$ & -8.49 & 16.86 & $48.06^{*}$ & 6.20 \\
\hline L20xT1 & $47.49 * *$ & 28.72 & 2.88 & 0.70 & 0.70 & 1.41 & 2.88 & 0.70 & 13.32 & $44.72 *$ & 22.29 & -12.29 \\
\hline L20xT2 & 23.67 & 7.93 & 0.72 & -1.41 & -0.70 & 0.00 & 0.72 & -1.41 & -21.04 & 0.84 & $49.66^{*}$ & 7.34 \\
\hline L21xT1 & 23.81 & 8.05 & $5.04 *$ & 2.82 & 2.10 & 2.82 & $5.04 *$ & 2.82 & -8.11 & 17.36 & 29.04 & -7.45 \\
\hline L21xT2 & 0.71 & -12.11 & 0.00 & -2.11 & -0.70 & 0.00 & 0.00 & -2.11 & -5.98 & 20.07 & 5.58 & -24.27 \\
\hline L22xT1 & 25.11 & 9.18 & $4.32 *$ & 2.11 & 2.10 & 2.82 & $4.32 *$ & 2.11 & -8.30 & 17.11 & 30.64 & -6.30 \\
\hline L22xT2 & 11.00 & -3.13 & $-4.32 *$ & $-6.34 * *$ & -4.90 & -4.23 & $-4.32 *$ & $-6.34 * *$ & -22.20 & -0.64 & 36.15 & -2.35 \\
\hline L23xT1 & 33.10 & 16.16 & 0.72 & -1.41 & -0.70 & 0.00 & 0.72 & -1.41 & -8.30 & 17.11 & $39.35^{*}$ & -0.05 \\
\hline L23xT2 & 24.75 & 8.87 & $-5.04 *$ & $-7.04 * *$ & -6.29 & -5.63 & $-5.04 *$ & $-7.04 * *$ & -11.20 & 13.41 & 33.84 & -4.00 \\
\hline L24xT1 & 7.26 & -6.40 & -2.88 & $-4.93 *$ & -5.59 & -4.93 & -2.88 & $-4.93 *$ & $-29.15^{*}$ & -9.52 & $45.40^{*}$ & 4.28 \\
\hline L24xT2 & 1.71 & -11.23 & $-6.47 * *$ & $-8.45^{* *}$ & -9.09 & -8.45 & $-6.47 * *$ & $-8.45 * *$ & $-37.07 * *$ & -19.63 & $54.28 * *$ & 10.66 \\
\hline L25xT1 & 12.08 & -2.19 & 0.72 & -1.41 & -0.70 & 0.00 & 0.72 & -1.41 & -14.86 & 8.73 & 25.31 & -10.12 \\
\hline L25xT2 & 23.38 & 7.68 & -2.88 & $-4.93 *$ & -4.20 & -3.52 & -2.88 & $-4.93 *$ & -20.66 & 1.33 & $48.24^{*}$ & 6.32 \\
\hline L26xT1 & 5.67 & -7.78 & 0.72 & -1.41 & -0.70 & 0.00 & 0.72 & -1.41 & -19.69 & 2.56 & 25.84 & -9.74 \\
\hline L26xT2 & 0.63 & -12.17 & -3.60 & $-5.63 * *$ & -4.90 & -4.23 & -3.60 & $-5.63 * *$ & $-39.19 * *$ & -22.34 & $57.31 * *$ & 12.83 \\
\hline L27xT1 & $39.22 *$ & 21.50 & 2.88 & 0.70 & 0.70 & 1.41 & 2.88 & 0.70 & -8.49 & 16.86 & $45.57 *$ & 4.41 \\
\hline L27xT2 & 8.19 & -5.58 & -2.16 & $-4.23 *$ & -2.80 & -2.11 & -2.16 & $-4.23 *$ & $-28.19^{*}$ & -8.28 & $43.44 *$ & 2.88 \\
\hline
\end{tabular}

Table 3. Continued...

\begin{tabular}{|c|c|c|c|c|c|c|c|c|c|c|c|c|}
\hline Code & GY & & DA & & DS & & DM & & BY & & $\mathrm{HI}$ & \\
\hline & BH546 & BH547 & BH546 & BH547 & BH546 & BH547 & BH546 & BH547 & BH546 & BH547 & BH546 & BH547 \\
\hline L28xT1 & 34.68 & 17.54 & 3.60 & 1.41 & 1.40 & 2.11 & 3.60 & 1.41 & -11.00 & 13.66 & $44.33 *$ & 3.52 \\
\hline L28xT2 & 3.87 & -9.35 & -2.16 & $-4.23^{*}$ & -4.90 & -4.23 & -2.16 & $-4.23 *$ & -23.36 & -2.12 & 27.98 & -8.21 \\
\hline L29xT1 & 2.65 & -10.42 & 0.72 & -1.41 & -0.70 & 0.00 & 0.72 & -1.41 & $-43.63 * *$ & -28.01 & $73.30 * *$ & 24.30 \\
\hline L29xT2 & 1.86 & -11.11 & -2.16 & $-4.23^{*}$ & -3.50 & -2.82 & -2.16 & $-4.23 *$ & $-44.21 * *$ & -28.75 & $74.19 * *$ & 24.94 \\
\hline L30xT1 & 18.99 & 3.84 & $5.76^{* *}$ & 3.52 & 2.80 & 3.52 & $5.76^{* *}$ & 3.52 & -10.42 & 14.40 & 26.73 & -9.10 \\
\hline L30xT2 & 29.07 & 12.64 & $4.32 *$ & 2.11 & 4.20 & 4.93 & $4.32 *$ & 2.11 & -6.95 & 18.84 & 31.89 & -5.41 \\
\hline L31xT1 & 4.09 & -9.16 & 2.16 & 0.00 & -0.70 & 0.00 & 2.16 & 0.00 & -20.08 & 2.07 & 24.07 & -11.01 \\
\hline L31xT2 & 22.73 & 7.11 & $-4.32 *$ & $-6.34 * *$ & -5.59 & -4.93 & $-4.32 *$ & $-6.34 * *$ & -19.11 & 3.30 & $45.22 *$ & 4.16 \\
\hline L32xT1 & -2.97 & -15.32 & $10.79 * *$ & $8.45^{* *}$ & $8.39 *$ & $9.15^{*}$ & $10.79 * *$ & $8.45 * *$ & 1.54 & 29.68 & -7.75 & $-33.83 *$ \\
\hline L32xT2 & -13.91 & -24.86 & $6.47 * *$ & $4.23^{*}$ & 5.59 & 6.34 & $6.47 * *$ & $4.23^{*}$ & $109.27^{* *}$ & $167.26^{* *}$ & -10.77 & $-36.00 * *$ \\
\hline SE(d) & 1.22 & 1.22 & 1.31 & 1.31 & 2.33 & 2.33 & 2.62 & 2.62 & 3.45 & 3.45 & 5.24 & 5.24 \\
\hline
\end{tabular}

$*$ and $* *=$ significant at 0.05 and 0.01 probability level, respectively $\mathrm{df}=$ degrees of freedom

$\mathrm{BY}=$ biomass yield, DA $=$ Days to Anthesis, DS = Days to Silking, DM = Days to Maturity, GY $=$ Grain Yield, $\mathrm{HI}=$ Harvest Index.

The extent of standard heterosis for ear length over BH546 varied from $9.90 \%$ for (L4xT2) to $-24.75 \%$ for (L32xT2). For this trait, except cross L4xT2 (9.90\%) and L9xT2 $(8.42 \%)$ that showed positive and significant standard heterosis, most of the crosses showed negative and significant to highly significant standard heterosis over BH546. In contrast to this, most of the crosses showed positive and significant to highly significant standard heterosis over BH547 except, cross L6xT1(-13.26\%), L32xT1(-12.71\%) and L32xT2 (-16.02\%) that showed negative and highly significant standard heterosis over BH547 for this trait. The extent of standard heterosis for ear length over BH547 varied from $22.65 \%$ for L4xT2 to $-16.02 \%$ for (L32xT2). In line with this finding, Gudeta (2007), Melkamu (2013) and Kumar et al. (2014) reported significant positive standard heterosis for ear length.

For ear diameter, standard heterosis over BH546 ranged from $4.21 \%$ for (L7xT2) to $-12.63 \%$ for (L3xT1). For this trait, 4 crosses showed positive and significant to highly significant standard heterosis over BH546 whereas 5 crosses exhibited negative and significant to highly significant standard heterosis over BH546. In contrast, all crosses showed negative and highly significant standard heterosis over BH547 except, cross L7xT1, 
L23xT1 and L31xT1 that showed negative and non- significant standard heterosis for this trait and ranged from $25.63 \%$ for (L3xT1) to $-7.71 \%$ for (L10xT1) and (L18xT1). For ear length and ear diameter, a cross with positive and significant standard heterosis was more advantageous as they increase the tendency of ear length and ear diameter than a standard check.

In case of number of ear per plant, standard heterosis over BH546 varied from $60.0 \%$ for (L3xT2) to $30.0 \%$ for nine (9) crosses including (L28xT1) and (L32xT1). For this trait, 18 crosses exhibited positive and significant to highly significant standard heterosis over BH546. Similarly, standard heterosis for this trait over BH547, nine (9) crosses showed positive and significant to highly significant standard heterosis and ranged from $45.45 \%$ for (L3xT2) to $27.27 \%$ for six (6) crosses including (L7xT1 and L17xT2). This indicating that increased in number of ears per plant for these crosses as compared to the standard checks.

For number of rows per ear, only cross L19xT1 (20.00\%) showed positive and significant standard heterosis over BH546 while cross L32xT2 (-20.00\%) showed negative and significant standard heterosis for the same trait. Similarly, 43 crosses showed negative and significant to highly significant standard heterosis over BH547 for this trait and ranged from $-29.41 \%$ for L32xT2 to $-11.76 \%$ for L18xT2, L19xT2 and L20xT1 (Table 3). In line with this finding, Gudeta (2007) Berhanu (2009) and shushay (2014) reported positive and significant heterosis and negative and highly significant standard heterosis.

The estimates of standard heterosis for number of kernels per row, cross L19xT1 (63.53\% and 73.32\%) showed positive and highly significant standard heterosis over both (BH546 and BH547) standard checks, respectively while crosses L32xT1 (-25.88\%) and L32xT2 (-35.29\%) showed negative significant and highly significant standard heterosis over BH546 respectively. Negative and significant standard heterosis for number of kernels per row over BH547 was exhibited by cross L32xT2 (-31.42\%).

For thousand kernel weight, nine (9) crosses exhibited positive and significant to highly significant standard heterosis over BH546 whereas 11 crosses exhibited positive and significant to highly significant standard heterosis over BH547. Cross L1xT2, L2xT2 and L31xT2 showed positive and highly significant $(\mathrm{P}<0.01)$ standard heterosis over both BH546 and BH547 whereas cross L3xT1 showed negative and significant $(\mathrm{P}<0.05)$ standard heterosis over both checks. The estimates of standard heterosis for this trait over both checks ranged from $44.81 \%$ for L $2 \times 12$ to $-17.64 \%$ for L3xT1. Positive and significant standard heterosis for thousand kernel weight was a desirable character in maize while negative and significant standard heterosis was undesirable character for this trait. In line with the result of the current finding, Gudeta (2007) in his study on heterosis and combining abilities in QPM versions of early generation highland maize inbred lines, reported high levels of heterosis over both parents for 1000-kernels weight. Similar results were reported by Melkamu (2013), Kumar et al. (2014) and shushay (2014). Table 3. Continued...

\begin{tabular}{|c|c|c|c|c|c|c|c|c|c|c|c|c|}
\hline Code & EL & & ED & & EPP & & RPE & & KPR & & TKW & \\
\hline & BH546 & BH547 & BH546 & BH547 & BH546 & BH547 & BH546 & BH547 & BH546 & BH547 & BH546 & BH547 \\
\hline L1xT1 & $-12.38 * *$ & -2.21 & 0.00 & $-14.87 * *$ & 20.0 & 9.09 & -6.67 & $-17.65^{*}$ & -15.29 & -10.22 & 12.22 & 12.22 \\
\hline L1xT2 & 0.99 & $12.71 * *$ & 2.11 & $-13.08 * *$ & 0.0 & -9.09 & -6.67 & $-17.65^{*}$ & -3.53 & 2.24 & $23.13 * *$ & $23.13^{* *}$ \\
\hline L2xT1 & $-13.86^{* *}$ & -3.87 & 4.21 & $-11.29 * *$ & 10.0 & 0.00 & -6.67 & $-17.65^{*}$ & -15.29 & -10.22 & 7.72 & 7.72 \\
\hline L2xT2 & 4.95 & $17.13^{* *}$ & 5.26 & $-10.39 * *$ & 20.0 & 9.09 & -6.67 & $-17.65^{*}$ & -4.71 & 1.00 & $44.81 * *$ & $44.81^{* *}$ \\
\hline L3xT1 & $-12.87 * *$ & -2.76 & $-12.63 * *$ & $-25.63 * *$ & $30.0^{*}$ & 18.18 & -6.67 & $-17.65^{*}$ & -5.88 & -0.25 & $-17.64 *$ & $-17.64 *$ \\
\hline L3xT2 & $-12.38 * *$ & -2.21 & 0.00 & $-14.87 * *$ & $60.0 * *$ & $45.45^{* *}$ & -13.33 & $-23.53 * *$ & -16.47 & -11.47 & 6.76 & 6.76 \\
\hline L4xT1 & -3.96 & 7.18 & 5.26 & $-10.39 * *$ & -10.0 & -18.18 & -6.67 & $-17.65^{*}$ & -3.53 & 2.24 & 8.26 & 8.26 \\
\hline L4xT2 & $9.90^{*}$ & $22.65 * *$ & -5.26 & $-19.35 * *$ & -10.0 & -18.18 & -13.33 & $-23.53 * *$ & -2.35 & 3.49 & 12.49 & 12.49 \\
\hline L5xT1 & -5.45 & 5.52 & 6.32 & $-9.50 * *$ & -10.0 & -18.18 & -6.67 & $-17.65^{* *}$ & -7.06 & -1.50 & 11.54 & 11.54 \\
\hline L5xT2 & 1.98 & $13.81^{* *}$ & -2.11 & $-16.67 * *$ & $30.0^{*}$ & 18.18 & -6.67 & $-17.65^{*}$ & -4.71 & 1.00 & 11.94 & 11.94 \\
\hline L6xT1 & $-22.28 * *$ & $-13.26^{* *}$ & 6.32 & $-9.50^{* *}$ & 0.0 & -9.09 & 0.00 & -11.76 & -18.82 & -13.97 & -12.05 & -12.05 \\
\hline L6xT2 & $-9.90 *$ & 0.55 & 6.32 & $-9.50 * *$ & 20.0 & 9.09 & -6.67 & $-17.65^{*}$ & -4.71 & 1.00 & 9.35 & 9.35 \\
\hline L7xT1 & -8.17 & 2.49 & 10.53 & -5.91 & $40.0^{* *}$ & $27.27 *$ & 0.00 & -11.76 & -1.18 & 4.74 & -2.78 & -2.78 \\
\hline L7xT2 & -7.43 & 3.31 & $4.21^{* *}$ & $-11.29 * *$ & 20.0 & 9.09 & -6.67 & $-17.65^{*}$ & -1.18 & 4.74 & -2.24 & -2.24 \\
\hline L8xT1 & 0.50 & $12.15^{*}$ & 7.37 & $-8.60^{* *}$ & 0.0 & -9.09 & -6.67 & $-17.65^{*}$ & 0.00 & 5.99 & 1.17 & 1.17 \\
\hline L8xT2 & -4.95 & 6.08 & $-2.11 *$ & $-16.67 * *$ & 20.0 & 9.09 & -6.67 & $-17.65^{*}$ & -8.24 & -2.74 & 3.63 & 3.63 \\
\hline L9xT1 & -4.95 & 6.08 & 5.26 & $-10.39 * *$ & 10.0 & 0.00 & 6.67 & -5.88 & -9.41 & -3.99 & 5.54 & 5.54 \\
\hline L9xT2 & $8.42 *$ & $20.99 * *$ & 2.11 & $-13.08 * *$ & -10.0 & -18.18 & -6.67 & $-17.65^{*}$ & 0.00 & 5.99 & 10.72 & 10.72 \\
\hline
\end{tabular}


Table 3. Continued...

\begin{tabular}{|c|c|c|c|c|c|c|c|c|c|c|c|c|}
\hline Code & EL & & ED & & EPP & & RPE & & KPR & & TKW & \\
\hline & BH546 & BH547 & BH546 & BH547 & BH546 & BH547 & BH546 & BH547 & BH546 & BH547 & BH546 & BH547 \\
\hline L10xT1 & -5.45 & 5.52 & 8.42 & $-7.71 *$ & $40.0 * *$ & $27.27 *$ & 6.67 & -5.88 & -4.71 & 1.00 & -5.92 & -5.92 \\
\hline L10xT2 & 5.94 & $18.23^{* *}$ & $-2.11^{*}$ & $-16.67 * *$ & 0.0 & -9.09 & -6.67 & $-17.65^{*}$ & -2.35 & 3.49 & 9.76 & 9.76 \\
\hline L11xT1 & $-8.42 *$ & 2.21 & 6.32 & $-9.50 * *$ & 0.0 & -9.09 & 0.00 & -11.76 & -3.53 & 2.24 & 13.04 & 13.04 \\
\hline L11xT2 & -6.93 & 3.87 & 2.11 & $-13.08 * *$ & 20.0 & 9.09 & 0.00 & -11.76 & -5.88 & -0.25 & $19.44 *$ & $19.44 *$ \\
\hline L12xT1 & -7.92 & 2.76 & 1.05 & $-13.98 * *$ & $40.0 * *$ & $27.27^{*}$ & 0.00 & -11.76 & -7.06 & -1.50 & -13.01 & -13.01 \\
\hline $\mathrm{L} 12 \times \mathrm{T} 2$ & -7.92 & 2.76 & -2.11 & $-16.67 * *$ & 20.0 & 9.09 & -6.67 & $-17.65^{*}$ & -1.18 & 4.74 & -4.69 & -4.69 \\
\hline L13xT1 & $-8.42 *$ & 2.21 & 6.32 & $-9.50 * *$ & $40.0 * *$ & $27.27^{*}$ & 0.00 & -11.76 & -18.82 & -13.97 & 1.31 & 1.31 \\
\hline L13xT2 & 0.99 & $12.71 * *$ & 2.11 & $-13.08 * *$ & 0.0 & -9.09 & -6.67 & $-17.65^{*}$ & -8.24 & -2.74 & $17.67^{*}$ & $17.67 *$ \\
\hline L14xT1 & $-9.90 *$ & 0.55 & 1.05 & $-13.98 * *$ & $40.0 * *$ & $27.27^{*}$ & -6.67 & $-17.65^{*}$ & -7.06 & -1.50 & 12.76 & 12.76 \\
\hline L14xT2 & $-8.42 *$ & 2.21 & -4.21 & $-18.46^{* *}$ & $30.0^{*}$ & 18.18 & -6.67 & $-17.65^{*}$ & -7.06 & -1.50 & -6.87 & -6.87 \\
\hline L15xT1 & 0.00 & $11.60^{*}$ & 10.53 & $-5.91 * *$ & 10.0 & 0.00 & -6.67 & $-17.65^{*}$ & 2.35 & 8.48 & 6.08 & 6.08 \\
\hline L15xT2 & -1.49 & 9.94* & $-3.16^{* *}$ & $-17.56 * *$ & 10.0 & 0.00 & -6.67 & $-17.65 *$ & 0.00 & 5.99 & 8.94 & 8.94 \\
\hline L16xT1 & -8.17 & 2.49 & 5.26 & $-10.39 * *$ & $30.0^{*}$ & 18.18 & 0.00 & -11.76 & -10.59 & -5.24 & -1.69 & -1.69 \\
\hline L16xT2 & -5.94 & 4.97 & 3.16 & $-12.19 * *$ & 10.0 & 0.00 & -6.67 & $-17.65^{*}$ & -1.18 & 4.74 & 16.03 & 16.03 \\
\hline L17xT1 & -7.92 & 2.76 & 4.21 & $-11.29 * *$ & $50.0 *$ & $36.36^{* *}$ & -6.67 & $-17.65^{*}$ & -7.06 & -1.50 & -1.55 & -1.55 \\
\hline L17xT2 & 1.49 & $13.26^{* * *}$ & -6.32 & $-20.25 * *$ & $40.0 * *$ & $27.27^{*}$ & -6.67 & $-17.65^{*}$ & -3.53 & 2.24 & 1.72 & 1.72 \\
\hline L18xT1 & -7.92 & 2.76 & 8.42 & $-7.71 *$ & 10.0 & 0.00 & 6.67 & -5.88 & -3.53 & 2.24 & -13.01 & -13.01 \\
\hline L18xT2 & -2.97 & 8.29 & $3.16^{*}$ & $-12.19 * *$ & 0.0 & -9.09 & 0.00 & -11.76 & -2.35 & 3.49 & 13.99 & 13.99 \\
\hline
\end{tabular}

Table 3. Continued...

\begin{tabular}{|c|c|c|c|c|c|c|c|c|c|c|c|c|}
\hline Code & EL & & ED & & EPP & & RPE & & KPR & & TKW & \\
\hline & BH546 & BH547 & BH546 & BH547 & BH546 & BH547 & BH546 & BH547 & BH546 & BH547 & BH546 & BH547 \\
\hline L19xT1 & -7.92 & 2.76 & 6.32 & $-9.50 * *$ & 20.0 & 9.09 & $20.00 *$ & 5.88 & $63.53^{* *}$ & $73.32^{* *}$ & -8.37 & -8.37 \\
\hline L19xT2 & 1.49 & $13.26^{* *}$ & 3.16 & $-12.19 * *$ & 10.0 & 0.00 & 0.00 & $-11.76^{*}$ & 0.00 & 5.99 & 1.58 & 1.58 \\
\hline L20xT1 & -6.44 & 4.42 & 6.32 & $-9.50^{* *}$ & $50.0^{* *}$ & $36.36^{*}$ & 0.00 & $-11.76^{*}$ & 0.00 & 5.99 & -2.78 & -2.78 \\
\hline L20xT2 & 2.48 & $14.36^{*}$ & 0.00 & $-14.87 * *$ & 10.0 & 0.00 & -6.67 & $-17.65^{*}$ & 2.35 & 8.48 & -4.42 & -4.42 \\
\hline L21xT1 & -6.93 & 3.87 & 6.32 & $-9.50 * *$ & $30.0^{*}$ & 18.18 & 6.67 & -5.88 & -1.18 & 4.74 & -0.60 & -0.60 \\
\hline L21xT2 & -0.99 & $10.50^{*}$ & 2.11 & $-13.08 * *$ & $30.0^{*}$ & 18.18 & 0.00 & -11.76 & -5.88 & -0.25 & -4.83 & -4.83 \\
\hline L22xT1 & $-8.91 *$ & 1.66 & 3.16 & $-12.19^{* *}$ & 20.0 & 9.09 & -6.67 & $-17.65^{*}$ & -14.12 & -8.98 & 9.35 & 9.35 \\
\hline L22xT2 & -6.68 & 4.14 & -3.16 & $-17.56^{* *}$ & 20.0 & 9.09 & -6.67 & $-17.65^{*}$ & -3.53 & 2.24 & -8.92 & -8.92 \\
\hline L23xT1 & -5.45 & 5.52 & 11.58 & -5.02 & 10.0 & 0.00 & 6.67 & -5.88 & 5.88 & 12.22 & -2.24 & -2.24 \\
\hline L23xT2 & -0.50 & $11.05^{* *}$ & $3.16^{* *}$ & $-12.19 * *$ & -10.0 & -18.18 & 0.00 & -11.76 & -1.18 & 4.74 & 4.04 & 4.04 \\
\hline L24xT1 & $-11.88^{* *}$ & -1.66 & 0.00 & $-14.87 * *$ & $30.0^{*}$ & 18.18 & 0.00 & -11.76 & -5.88 & -0.25 & -16.01 & -16.01 \\
\hline L24xT2 & -3.47 & 7.73 & -5.26 & $-19.35^{* *}$ & 0.0 & -9.09 & -6.67 & $-17.65^{*}$ & -4.71 & 1.00 & 16.72 & $16.72 *$ \\
\hline L25xT1 & -0.99 & $10.50^{* *}$ & 3.16 & $-12.19 * *$ & 10.0 & 0.00 & 0.00 & -11.76 & 1.18 & 7.23 & -2.37 & -2.37 \\
\hline L25xT2 & 4.95 & $17.13^{* *}$ & -1.05 & $-15.77 * *$ & 10.0 & 0.00 & -6.67 & $-17.65 *$ & -2.35 & 3.49 & 17.40 & $17.40 *$ \\
\hline L26xT1 & 0.00 & $11.60^{*}$ & 5.26 & $-10.39 * *$ & 10.0 & 0.00 & 6.67 & -5.88 & -7.06 & -1.50 & -5.37 & -5.37 \\
\hline L26xT2 & -7.92 & 2.76 & -6.32 & $-20.25 * *$ & 10.0 & 0.00 & -6.67 & $-17.65^{*}$ & -9.41 & -3.99 & -12.60 & -12.60 \\
\hline L27xT1 & -0.99 & $10.50^{*}$ & 3.16 & $-12.19 * *$ & 0.0 & -9.09 & 0.00 & -11.76 & 1.18 & 7.23 & -8.78 & -8.78 \\
\hline L27xT2 & -3.47 & 7.73 & -6.32 & $-20.25^{* *}$ & 10.0 & 0.00 & -6.67 & $-17.65^{*}$ & -5.88 & -0.25 & -3.60 & -3.60 \\
\hline
\end{tabular}

Table 3. Continued...

\begin{tabular}{|c|c|c|c|c|c|c|c|c|c|c|c|c|}
\hline Code & EL & & ED & & EPP & & RPE & & KPR & & TKW & \\
\hline & BH546 & BH547 & BH546 & BH547 & BH546 & BH547 & BH546 & BH547 & BH546 & BH547 & BH546 & BH547 \\
\hline L28xT1 & $-9.90 *$ & 0.55 & -4.21 & $-18.46^{* *}$ & $30.0^{*}$ & 18.18 & -6.67 & $-17.65^{*}$ & -10.59 & -5.24 & -4.96 & -4.96 \\
\hline L28xT2 & -0.99 & $10.50 *$ & -3.16 & $-17.56^{* *}$ & 20.0 & 9.09 & -6.67 & $-17.65^{*}$ & 1.18 & 7.23 & 9.63 & 9.63 \\
\hline L29xT1 & -11.39 & -1.10 & -2.11 & $-16.67 * *$ & 20.0 & 9.09 & -6.67 & $-17.65^{*}$ & -10.59 & -5.24 & -11.92 & -11.92 \\
\hline L29xT2 & $-10.40^{*}$ & 0.00 & -3.16 & $-17.56 * *$ & 10.0 & 0.00 & -6.67 & $-17.65^{*}$ & -15.29 & -10.22 & 7.04 & 7.04 \\
\hline L30xT1 & -0.99 & $10.50 *$ & 13.68 & $-3.23 * *$ & 0.0 & -9.09 & -6.67 & $-17.65^{*}$ & -1.18 & 4.74 & $19.03 *$ & $19.03 *$ \\
\hline L30xT2 & -0.99 & $10.50 *$ & $-2.11 * *$ & $-16.67 * *$ & 10.0 & 0.00 & -6.67 & $-17.65^{* *}$ & -5.88 & -0.25 & $19.17 *$ & $19.17^{*}$ \\
\hline L31xT1 & $-12.38 * *$ & -2.21 & 8.42 & -7.71 & 10.0 & 0.00 & -6.67 & $-17.65^{*}$ & -8.24 & -2.74 & -7.14 & -7.14 \\
\hline L31xT2 & $-10.89 * *$ & -0.55 & $3.16^{*}$ & $-12.19 * *$ & 10.0 & 0.00 & 0.00 & -11.76 & -10.59 & -5.24 & $28.17 * *$ & $28.17 * *$ \\
\hline L32xT1 & $-21.78^{* *}$ & $-12.71 * *$ & -1.05 & $-15.77 * *$ & $30.0^{*}$ & 18.18 & -6.67 & $-17.65^{*}$ & $-25.88^{*}$ & -21.45 & 5.81 & 5.81 \\
\hline L32xT2 & $-24.75 * *$ & $-16.02 * *$ & -4.21 & $-18.46 * *$ & 20.0 & 9.09 & $-20.00^{*}$ & $-29.41 * *$ & $-35.29 * *$ & $-31.42 *$ & $41.67 * *$ & $41.67 * *$ \\
\hline SE(d) & 0.84 & 0.84 & 0.17 & 0.17 & 0.13 & 0.13 & 1.41 & 1.41 & 5.05 & 5.05 & 29.74 & 29.74 \\
\hline
\end{tabular}

$*$ and $* *=$ significant at 0.05 and 0.01 probability level, respectively $\mathrm{df}=$ degrees of freedom

$\mathrm{EL}=$ Ear Length, $\mathrm{ED}=$ Ear Diameter, $\mathrm{EPP}=$ Number of Ears per Plant, KPR $=$ Number of Kernels per row, $\mathrm{RPE}$ $=$ Number of Kernel Rows per Ear, TKW $=$ Thousand Kernel Weight.

\section{CONCLUSION}

In this study, considerable standard heterosis for all traits over both commercial checks was manifested. The highest standard heterosis for grain yield observed from L17xT1 (61.75 \%) and (41.46\%) over BH546 and BH547, respectively. This indicated that, the presence of exploitable heterosis essential for this trait. Cross L3xT2 manifested negative standard heterosis over BH546 and BH547 for days to anthesis, days to silking and days to maturity that indicates earliness in maturity. Similarly, most of the crosses showed positive standard heterosis for biomass yield, harvest index, ear per plant, ear length, ear diameter, rows per ear, kernel per rows and thousand kernel weights over both commercial checks.

The existence of genetic variation for grain yield and all yield related traits give further direction for maize breeders those who are interested in heterosis breeding. However, further evaluation at more locations and over years is advisable to confirm the promising results observed in present study. 


\section{ACKNOWLEDGEMENT}

I would like to pay my sincere gratitude to the Ethiopian Institute of Agricultural Research (EIAR) for giving me the financial support required to do the research work and for all other rounded support. Great thanks owed to Haramaya University for educating me towards my MSc degree. The staff members of Bako National maize and Hawassa maize research section are highly acknowledge for their valuable assistance and immense contributions in executing and handling the field experiment and cooperation in data collection.

\section{REFERENCES}

Berhanu Tadesse. (2009). 'Heterosis and combining ability for yield, yield related parameters and stover quality traits for food-feed in maize (Zea mays L.) Adapted to the midaltitude agro- ecology of Ethiopia', MSc Thesis School of Graduate studies, Haramaya University, Ethiopia.

Chandana .B.C. (2013). 'Heterosis and combining ability studies in maize (Zea mays L.) with emphasis on Turcicum leaf blight [Exserohilumturcicum. MSc Thesis Presented to College of Agriculture, Dharwad University of agricultural sciences, Dharwad.

CSA. (2018). Agricultural Sample survey: Report on area and production of major crops (private peasant holdings, Meher season). Statistical Bulletin, (1).Addis Abeba

Falconer, D.S. and T.F.C.Mackay.(1996). Introduction to Quantitative Genetics. $4^{\text {th }}$ ed. Longman, London.

FAO (Food and Agriculture Organization of the United Nations). (2016). Crop Prospects and Food Situation

FAOSTAT. (2016). Statistical databases and data-sets of the Food and Agriculture Organization of the United Nations.

Gudata Napir. (2007). Heterosis and combining abilities in QPM varieties of early generation high land maize (Zea mays L.) Inbred lines. M.Sc. Thesis. Haramaya University of Agriculture, School of Graduate Studies. Haramaya, Ethiopia.185.

Kumar, G. P., Prashanth, Y., Reddy, V. N., Kumar, S. S. and Rao, P. V. (2014). 'International Journal of Pure \& Applied Bioscience Heterosis for Grain yield and its Component traits in Maize ', 2(1): 106-111.

Melkamu. (2013). Estimation of combining ability and heterosis of quality protein maize inbred lines. African Journal of Agricultural, 8(48): 6309-6317.

Mesenbet Z., Zeleke Habtamu and Wolde Leggase. (2016). 'Standard heterosis of pipeline maize hybrids for grain yield and yield related traits at Pawe, North western Ethiopia', 3(2).

Mosisa Worku.,Wolde Legesse, Tadesse Berhanu, Demisse Girma, Azmach Girum, AberaWende, Keno Tolera, Bogale Gezahegn, Wegary Dagne, Admasu Solomon, Zell eke Habtamu, Y. Kassa, Chibsa Temesgen, J. Habte, Negara Demoz, and B. Getachew. (2012). Status and future direction of maize research and production in Ethiopia. 17-23. In Mosisa et al (ed.) Proceedings of the Third National Maize Workshop of Ethiopia, Addis Ababa, Ethiopia. 16-20 April 2012. EIAR and CIMMYT, Addis Ababa, Ethiopia.

SAS. (2004). Statistical Analysis System, User's Guide. Statistical. Version $7^{\text {th }}$ ed. SAS. Inst. Inc. Cary. N.C. USA. Schnell FW.(1982). A synoptic study of the methods and categories of plant breeding. Pflanzenzüchtung.89: 1-18. Shushay Welderufael. (2014). Standard Heterosis of Maize (Zea mays L.) Inbred Lines for Grain Yield and Yield Related Traits in Central Rift Valley of Ethiopia. Journal of Biology, Agriculture and Healthcare. 4 (23): 2224-3208 